\title{
A new approach to automatic continuous artery diameter measurement
}

\author{
Bartosz Zieliński and Adam Roman \\ Institute of Computer Science \\ and Computer Mathematics, \\ Faculty of Mathematics and Computer Science, \\ Jagiellonian University, \\ ul. Łojasiewicza 6, 30-348 Kraków, Poland \\ Email: \{bartosz.zielinski, adam.roman\}@uj.edu.pl
}

\begin{abstract}
In this paper, we present an application which aid an evaluation of the arterial diameter changes, based on ultrasound videos. The designed, implemented and verified algorithm uses the techniques of image processing, image analysis and pattern recognition, such as filtering, profile plot analysis and active contour method. Except determining the artery diameter over time it is also able to retrieve ECG from ultrasound video. The results obtained for both signals are synchronized, therefore it is possible to obtain the artery diameters in $R$ wave points, which is a novel approach. Experiments were performed to assess the software validation by comparing the outcomes obtained with the evaluated algorithm with those manually-acquired the correlation is high. This is the first stage of the research in which we will build the cardiovascular predictive model to search for the new cardiovascular factors.
\end{abstract}

\section{INTRODUCTION}

I NCREASED risk of cardiovascular diseases is related to endothelial dysfunction, which can be assessed based on the ultrasonic monitoring of brachial artery diameter changes in response to the temporal closure of the vessel. During the examination, videos containing the brachial artery representation in B-mode and M-mode are simultaneously captured (left and right side in Fig. 1). B-mode is a 2D image of the brachial artery in the longitudinal plane. A single scan line placed along B-mode is used by ultrasound device to generate M-mode representation, which describes how the structures intersect with that line in time.

Manual measurement performed on such videos is operatordependent, prone to mistakes and time-consuming. Moreover, it is impossible to perform continuous measurement manually, due to frames quantity (30 frames per second).

In this paper, we present an application which can aid an evaluation of the arterial diameter changes, based on ultrasound videos. The designed, implemented and verified algorithm uses the techniques of image processing, image analysis and pattern recognition, such as profile plot analysis and active contour method [9]. Except determining the artery diameter over time it is also able to retrieve ECG from an M-mode ultrasound. The results obtained for both signals are synchronized, therefore it is possible to obtain the artery diameters in $\mathrm{R}$ wave points, which is a novel approach introduced

\author{
Agata Dróżdż, Agata Kowalewska, \\ and Marzena Frołow \\ Jagiellonian Centre for Experimental Therapeutics, \\ Jagiellonian University, \\ ul. Bobrzyńskiego 14, 30-348 Kraków, Poland \\ Email: \{agata.drozdz, agata.kowalewska, \\ marzena.frolow $\} @$ jcet.eu
}

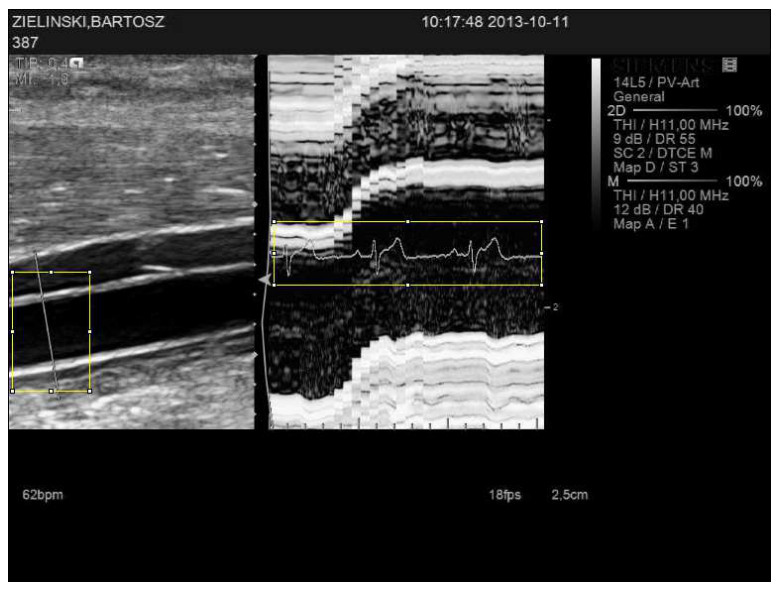

Fig. 1. The example of the first frame from the ultrasound video with B-mode and M-mode on the left and right side, respectively.

in our algorithm.

The test set includes baseline, occlusion and post-deflation videos and images obtained from 20 patients, together with the automatically and manually-measured values. Such test set was used to conduct analyses, results of which were investigated.

\section{THE MEDICAL ASPECTS OF THE PAPER}

The endothelium, forming the innermost, unicellular layer of arteries, plays a key role in the homeostasis of blood vessels. Properly functioning endothelial cells, in response to shear stress (SS) variations produce certain particles that may cause dilation or constriction of the vessel. Increased SS results in releasing vasodilators such as Endothelium-Derived Relaxing Faxtor (Nitric Oxide), Endothelium-Derived Hyperpolarizating Factor and prostacyclin, causing a local increase in the vessel diameter. Disorders in the excretion of these substances can be associated with the early endothelial dysfunction, which among others is related to increased likelihood of cardiovascular diseases [2], [7].

One of the most commonly used method for non-invasive assessment of endothelial function is the flow mediated- 
a)

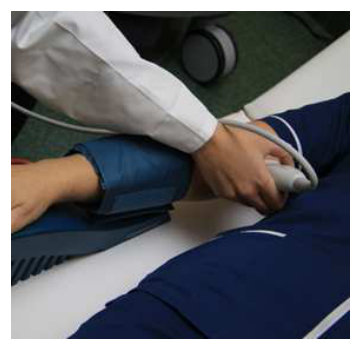

b)

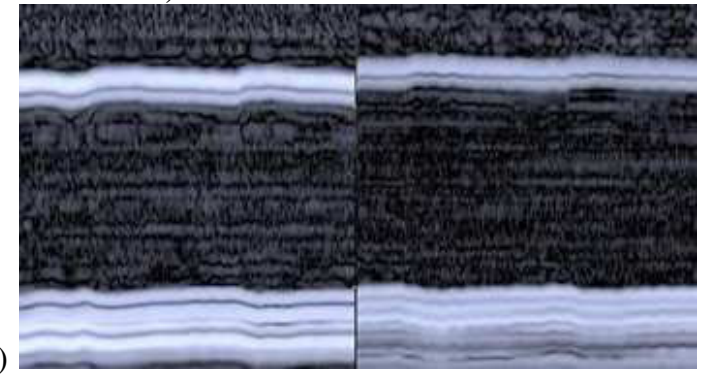

Fig. 2. Cuff and ultrasonic probe placement - a. M-modes of the arterial baseline (left side) and its dilation after releasing the cuff (right side) - b.

dilation (FMD) study. It is based on the ultrasonic monitoring of the brachial artery diameter changes in response to the temporal closure of the vessel (ischemia). Artery closure is achieved through the pressure cuff inflation on the limb (see Fig. 2a). After releasing the cuff the shear stress acting on the endothelial cells in the vessel wall is augmented as a result of increased flow, causing a reactive hyperemia [3]. The arterial diameter varies between 2 and $5 \mathrm{~mm}$ and its change ranges from 0 to $25 \%$ (see Fig. 2b). The accurate calculation of such a small variation would be difficult due to the limitations of the standard USG, therefore artery imaging is performed using a high frequency ultrasound probe (7-14 MHz).

\section{THE EXISTING METHODS}

Most of the FMD measurements in the literature are done by tracing the vessel boundary manually. Such a process is time consuming and error-prone. Therefore, several authors proposed some automated methods for this task. In [5] the authors proposed a robust automated measurement of the vasodilator response by automatically locating the artery using a variable window method and global constraint deformable model for vessel wall boundary detection. Geminiani et al. [6] used a robust edge detection algorithm, called "mass center of the gray level variability". Faita et al. [4] introduced the localization algorithm of the artery tunics based on a new mathematical operator called the first order absolute moment and on a pattern recognition approach. Bartoli et al. [1] utilized a spline model (deformable template) that detects the artery boundaries and track them all along the video sequence. The a priori knowledge about the image features and its content is exploited in this approach. Kaneko et al. [8] developed a method of measuring the change in the thickness and the elasticity of the brachial artery during a cardiac cycle using the so-called phased tracking method for the evaluation of the mechanical property of only the intima media region.

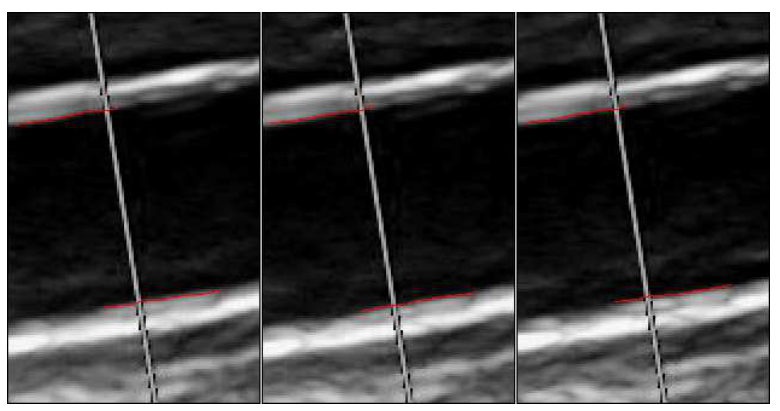

Fig. 3. The successive steps of the artery diameter analysis.

Our approach is based on profile plot analysis and active contour method. As opposed to the existing models, our algorithm, except determining the artery diameter over time, also retrieves ECG from $\mathrm{M}$-mode and is resistant to the additional line in B-mode. The existing commercial medical applications for FMD measurement, such as Quipu FMD Studio $^{1}$, or MIA Vascular Tools $5^{2}$ do not return the partial results like continues artery diameter. On the other hand, such partial product is necessary to conduct medical research. Other algorithms are sold only with the new machinery, like Ultrasonix products ${ }^{3}$. Our approach does not depend on any machinery.

\section{THE ALGORITHM}

The presented algorithm can be employed for artery diameter measurement and FMD study. It is a semi-automatic technique, which operates on the basis of two regions of interest (ROI) selected by the operator in the first frame of the ultrasound video (see Fig. 1). First ROI contains a fragment of B-mode with scan line (left yellow rectangle in Fig. 1). The ECG representation is selected as the second ROI (right yellow rectangle in Fig. 1).

The first ROI is given together with the initial outlines of the arterial walls, which are automatically tracked at the following frames of the video (see Fig. 3). The second region of interest is analysed fully automatically.

\section{A. Determining the artery diameter over time}

The algorithm for determining the artery diameter over time works for both outlines independently and is based on the active contour method [9]. In both cases, it starts with the outline containing 2 points (beginning and end of the section given by the system operator), which is decomposed to obtain series of 10 points with the same distance between successive elements.

Representing the position of a snake parametrically by $\mathrm{v}(\mathrm{s})$, we can write its energy functional as [9]:

$E_{\text {snake }}^{*}(v)=\int E_{\text {int }}(v(s))+E_{\text {image }}(v(s))+E_{\text {con }}(v(s)) d x$.

\footnotetext{
${ }^{1}$ http://www.quipu.eu/fmd.php

${ }^{2}$ http://www.mia-llc.com/products/vascular_fda.htm

${ }^{3} \mathrm{http}: / /$ www.ultrasonix.com/research/clinical
} 


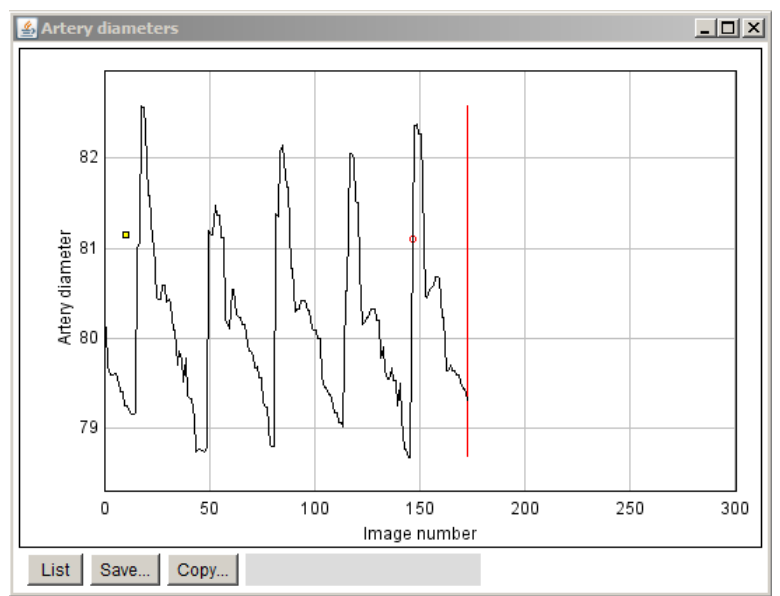

Fig. 4. The partial result of the artery diameter analysis.

Internal spline energy contains a first-order term controlled by $\alpha$, and a second-order term controlled by $\beta$ :

$$
E_{\text {int }}(v(s))=\frac{\alpha\left|v_{s}(s)\right|^{2}+\beta\left|v_{s s}(s)\right|^{2}}{2},
$$

The first-order term is responsible for stretching and the second-order is responsible for bending. The values of both factors were initially set to 0.5 , to prevent extensive stretching and bending. Those preliminary values proved to be accurate (see Section VI) and therefore were not explored more specifically, but we are plan to do it in the future.

The total image energy can be exposed as a weighted combination of the three energy functionals:

$$
E_{\text {image }}(v(s))=w_{\text {line }} E_{\text {line }}+w_{\text {edge }} E_{\text {edge }}+w_{\text {term }} E_{\text {term }},
$$

$E_{\text {line }}=I$ is the image intensity itself, $E_{\text {edge }}=-|\nabla I|^{2}$, and $E_{\text {term }}$ is terminal functional utilized in order to find terminations of line segments and corners. The value of $w_{\text {edge }}$ was set to 1 to promote the edges, while both other factors were set to 0 , not to promote darker or lighter regions. Moreover, the Gaussian blur is applied to an image before computing $\nabla I$ and then it is scaled to $[0,1]$. With reference to such approach, the factor $\gamma$ responsible for step size was set to 0 .

Functional $E_{c o n v}$ responsible for external constrain forces was currently omitted, but we plan to use it in the feature to promote parallelism between both outlines.

The endpoints $\left(P_{1}\right.$ and $\left.P_{10}\right)$ are treated differently than the other points. Their x coordinate left unchanged, while their $y$ coordinates are obtained using linear approximation computed for the outline. It is necessary, because otherwise the outlines would shrink into two points. In the last step of the iteration, each outline is modified so that the distance between the successive points is the same and successive iteration begins.

Active contour method works as long as distance between the outlines from the successive iterations is bigger than $0.000001 \mathrm{~mm}$. As the result of such analysis for each frame, the sequence of the artery diameters is obtained (see Fig. 4).

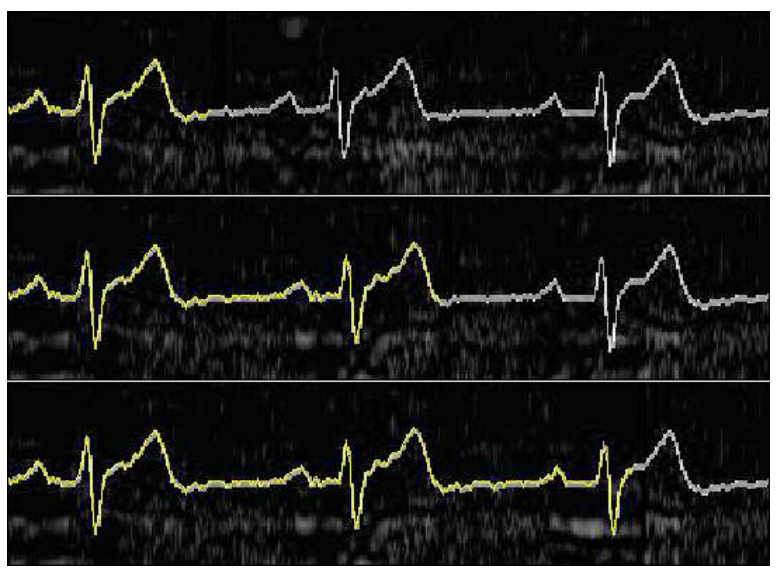

Fig. 5. The successive steps of the ECG retrieval analysis.

\section{B. ECG retrieval from $M$-mode}

The algorithm for ECG retrieval is based on M-mode which is cyclic (see Fig. 1). Therefore, in order to retrieve ECG it finds the most differing columns of two successive video frames. For each of them the profile plot is generated and then analysed to determine the pixels with the maximal values. Thanks to such approach, the analysis is invariant to noise and equipment type (see Fig. 5).

\section{EXPERIMENTS}

The software validation was performed by comparing the outcomes obtained with the evaluated algorithm with those manually-acquired. The test procedure is as follows. Before the test a pressure cuff is placed on the patientâĂn̆s forearm and the 14-MHz ultrasonic transducer is placed on the upper arm (proximal to the cuff), fixed on a tripod. During the whole test videos containing the brachial artery representation in Bmode and M-mode are simultaneously captured (Fig. 1). A baseline video is recorded for 10 seconds. Thereafter arterial occlusion is created by inflating the cuff for 5 minutes to about $50 \mathrm{mmHg}$ above patientâĂňs systolic pressure. The last, 60second video is recorded continuously from 5 th second after the cuff deflation.

Hence, there are 7 points in time where the measurements are taken: baseline (called "pre" in the following graphs), arterial occlusion ("4min") and 5 points in time after cuff deflation (in 20th, 30th, 40th, 50th and 60th second). The software tracks the wall borders using B-mode video representation and it displays arterial diameter changing in time as a result. After a calibration it is possible to determine vesselâĂňs peak value. The manual diameter measurements were performed using M-mode images captured every 10 seconds after the cuff deflation, starting from the fifth second. The diameter was measured in each image at $\mathrm{R}$ wave points (an average from three measurements).

The data come from the tests (both manual and automatic) done for 20 patients. Fig. 6a shows the averaged sequence of measurements for both approaches. The results for the baseline, arterial occlusion and 30s are almost identical. Both 

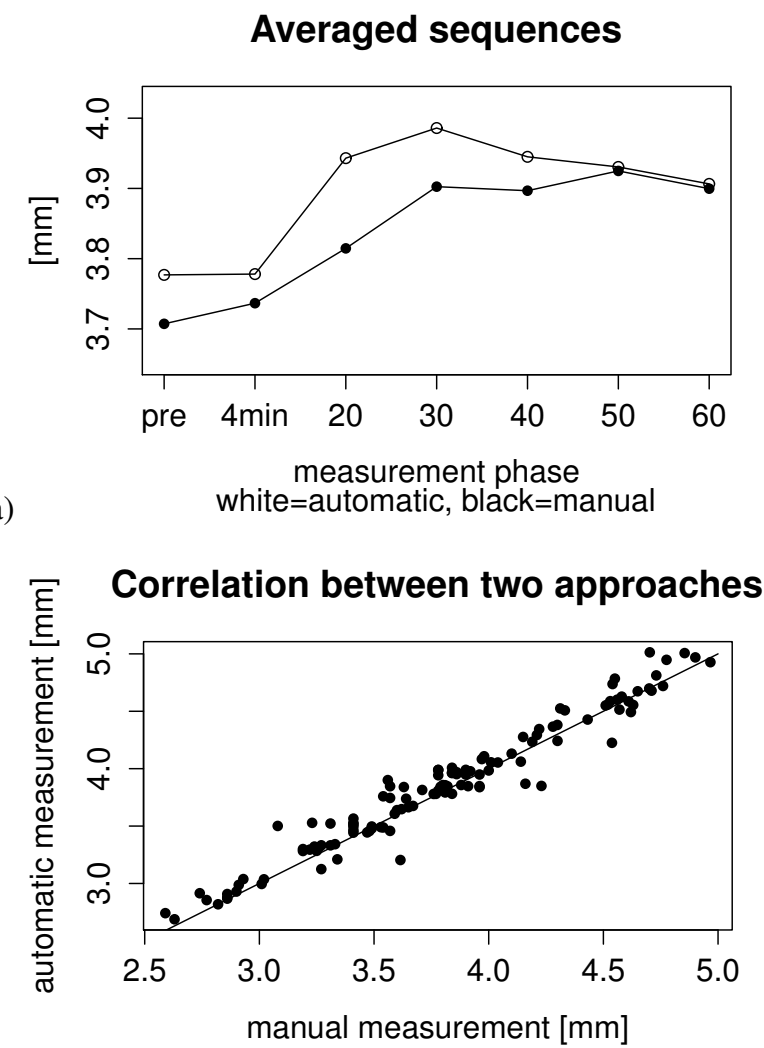

b)

Fig. 6. Averaged manually and automatically obtained sequences - a Correlation between manual and automatic measurements - $b$.

approaches give similar results also for 40,50 and 60 s. For 20s point we can see the larger difference between manual and automatic approach. This is due to the problems with manual measurements mentioned earlier - they are operatordependent and more prone to mistakes. There is also a threat of missing the right value due to 10 second intervals between measurements. In the initial phase the ultrasonic transducer can be still tuned to capture the good quality image of the artery. As one can see from Fig. 6a, automatic method works better, because the pressure in the 20th second after cuff release should be much higher than it follows from the manual measurement. The automatic measurements follow the right scheme here.

Correlation between manual and automatic measurements is shown in Fig. 6b. Each point in this figure represents a measurement from one phase (baseline, cuff occlusion, 20s, $30 \mathrm{~s}, 40 \mathrm{~s}, 50 \mathrm{~s}$ or $60 \mathrm{~s}$ ) for one patient. The correlation is 0.98 . This means that in general the automatic method is well aligned with the manual one, so we can claim that the method is accurate. The points lying farther from the $y=x$ line refer mainly to the situation from 20th second presented in Fig. 6a, where the operator can make an inaccurate measurement (this will be one of the topic of the future research). The mean difference between manual and automatic measurement was
TABLE I

DIFFERENCE BETWEEN MANUAL AND AUTOMATIC MEASUREMENTS SPLIT BY PHASE (IN MILLIMETRES).

\begin{tabular}{|r|c|c|c|c|c|c|c|}
\hline phase & pre & 4min & $\mathbf{2 0}$ & $\mathbf{3 0}$ & $\mathbf{4 0}$ & $\mathbf{5 0}$ & $\mathbf{6 0}$ \\
\hline \hline diff & -0.13 & -0.09 & -0.19 & -0.15 & -0.11 & -0.07 & -0.006 \\
stddev & 0.56 & 0.26 & 0.34 & 0.34 & 0.27 & 0.19 & 0.08 \\
\hline
\end{tabular}

$-0.055 \mathrm{~mm}$. The mean differences split by phase is shown in Table I.

The automatic measurements are slightly larger than the manual ones. This is due to the fact that the operator might use her own method of marking the diameter, with endpoints lying closer to each other than in the automatic case. Hence, in case of the manual measurement there may be a systematic error. However, this doesn't matter, as the main metric used in the subsequent analysis is the FMD, defined by the formula $F M D=\frac{p d h-b}{b} \cdot 100 \%$, where $p d h$ is a peak diameter in hyperemia and $b$ is a baseline value. The FMD is a ratiotype metric, so systematic error doesn't affect the differences between automatic and manual measurements given in terms of FMD.

\section{CONCLUDING REMARKS}

In this paper we presented an application for monitoring the changes in arterial diameter, based on ultrasound videos. It was validated by comparing its outcomes with those acquired manually. The tool turned out to be very accurate and outperforms manual measurement process. Except determining the artery diameter over time it is also able to retrieve ECG from an M-mode ultrasound. The results obtained for both signals are synchronized, therefore it is possible to obtain the artery diameters in $\mathrm{R}$ wave points, which is a novel approach introduced in our algorithm (see Fig. 7).

This is the first stage of the research in which we will build the cardiovascular predictive model to search for the new cardiovascular factors. Therefore, the following will be introduced during the further part of the work. First, both regions of interest shown in Fig. 1 will be selected automatically, together with the initial outlines of the arterial walls (see Fig. 3 ). Second, we will quantitatively describe the artery diameter over time in terms of its characteristic components, such as the slope of its initial part and length of the plateau. As the result, the software will be used to evaluate a distensibility of arterial wall (e.g. carotid).

\section{REFERENCES}

[1] Bartoli G. et al. 2008. Model-based analysis of flow-mediated dilation and intima-media thickness. J Biomed Imaging 16, http://dx.doi.org/10.1155/2008/738545

[2] Celermajer D.S. 1997. Endothelial dysfunction: does it matter? Is it reversible? J Am Coll Cardiol 30:325-333, http://dx.doi.org/10.1016/S0735-1097(97)00189-7

[3] Dick H.J. et al. 2011. Assessment of flow-mediated dilation in humans: a methodological and physiological guideline. Am J Physiol-Heart Circ Physiol 300:H2-H12, http://dx.doi.org/10.1152/ajpheart.00471.2010

[4] Faita F. et al. 2008. Detection of artery interfaces: a real-time system and its clinical applications. Med Imaging 69200F-69200F, http://dx.doi.org/10.1117/12.770408 


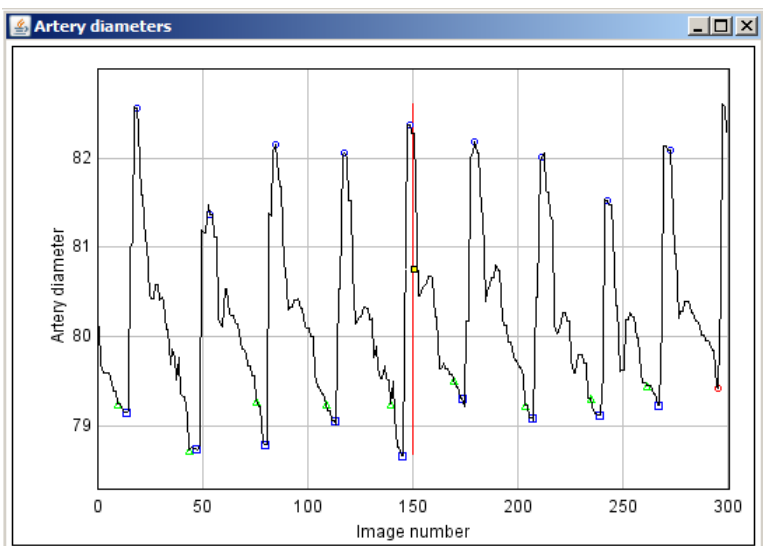

a)

List Save... Copy... $\mathrm{X}=296.3, \mathrm{Y}=80.5$

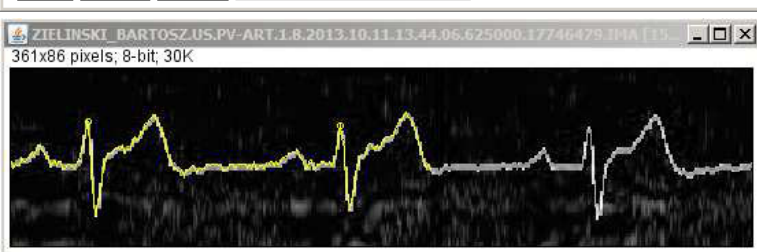

b)

Fig. 7. The result of the artery diameter and ECG analysis - a and b, respectively. The $\mathrm{R}$ wave points are marked as green triangles in a and yellow circles in $\mathrm{b}$. Blue circles and squares in a are local maximums and minimums of the artery diameter.
[5] Fan L. et al. 2000. Ultrasound measurement of brachial flowmediated vasodilator response. IEEE Trans Med Imaging 19:621-631, http://dx.doi.org/10.1109/42.870669

[6] Gemignani V. et al. 2007. A system for real-time measurement of the brachial artery diameter in B-mode ultrasound images. IEEE Trans Med Imaging 26:393-404, http://dx.doi.org/10.1109/TMI.2006.891477

[7] Jazuli F., Pyke K.E.. 2011. The impact of baseline artery diameter on flow-mediated vasodilation: a comparison of brachial and radial artery responses to matched levels of shear stress. Am J Physiol-Heart Circ Physiol 301:H1667-H1677, http://dx.doi.org/10.1152/ajpheart.00487.2011

[8] Kaneko T. et al. 2007. Ultrasonic measurement of change in elasticity due to endothelium dependent relaxation response by accurate detection of artery-wall boundary. Jpn J Appl Phys 46:4881, http://dx.doi.org/10.1143/JJAP.46.4881

[9] Kass M. et al. 1988. Snakes: Active contour models. Int J Comput Vision $1: 321-331$ 